\title{
Paeoniflorin attenuates hippocampal damage in a rat model of vascular dementia
}

\author{
YING ZHANG $^{1}$, LI-LI WANG ${ }^{2}$, YAN WU $^{3}$, NING WANG ${ }^{4}$, SHANG-MING WANG $^{4}$, \\ BIN ZHANG ${ }^{4}$, CUI-GE SHI ${ }^{4}$ and SHU-CHENG ZHANG ${ }^{4}$
}

\begin{abstract}
${ }^{1}$ Research Center of Biomaterial and Novel Drug Delivery Systems, National Research Institute of Family Planning, Beijing 100081; ${ }^{2}$ Chinese Academy of Medical Sciences and Peking Union Medical College, Beijing 100050; ${ }^{3}$ Department of Neurobiology, Beijing Institute of Basic Medical Sciences, Beijing 100850;

${ }^{4}$ Department of Cell Biology, National Research Institute of Family Planning, Beijing 100081, P.R. China
\end{abstract}

Received March 12, 2015; Accepted April 14, 2016

DOI: 10.3892/etm.2016.3849

\begin{abstract}
Paeoniflorn(PF), the principal bioactive component of Paeoniae radix prescribed in traditional Chinese medicine, possesses a wide range of biological effects and exhibits neuroprotective effects in numerous diseases. Previous studies have demonstrated that PF significantly attenuates memory impairment in rats with vascular dementia (VD). In the present study, a bilateral common carotid artery occlusion (BCCAO) rat model was used to explore the underlying mechanisms of PF. The expression levels of neuron-specific enolase (NSE), S100 $\beta$, B-cell lymphoma 2 (Bcl-2), Bcl-2 associated $\mathrm{X}$ protein, cytochrome $c$ and brain-derived neurotrophic factor (BDNF) in the hippocampus were measured by western blot analysis. The results showed that administration of PF for 28 days significantly decreased the expression levels of NSE and S100 $\beta$, both sensitive markers for brain damage, in vascular dementia (VD) model rats. In addition, $\mathrm{PF}$ inhibited the initiation of apoptotic cell death and attenuated the decreased expression levels of BDNF induced by bilateral common carotid artery occlusion. These data confirm the neuroprotective effects of PF on VD and provide a novel insight into the long-term use of PF as a potential treatment in the stages of early cognitive impairment in VD.
\end{abstract}

\section{Introduction}

Vascular dementia (VD) is the second most common type of dementia after Alzheimer's disease $(1,2)$. It is predicted that by the year 2040, the number of people with dementia

Correspondence to: Professor Cui-Ge Shi or Professor Shu-Cheng Zhang, Department of Cell Biology, National Research Institute of Family Planning, 12 Dahuisi Road, Beijing 100081, P.R. China

E-mail: yushuiyushui@sina.com

E-mail:nrifp@263.net

Key words: paeoniflorin, vascular dementia, hippocampus, apoptosis worldwide will reach 81.1 million, with the number of dementia patients in China being the sum of all those in all the developed countries combined (3). The progressive nature of VD leads to an unremitting and largely irreversible deterioration in quality of life, and places a heavy emotional and economic burden on families (4). However, currently there are no drugs licensed for the treatment of vascular cognitive impairment. Thus, there is an urgent requirement to develop therapeutic agents to prevent vascular dementia.

VD is caused by a reduced blood flow to the brain or an impaired vascular system (5). As the population ages and cerebrovascular disease prevalence increases, the occurrence of VD increases as well (6). Etiopathogenic mechanisms causing VD include oxidative stress, cytotoxicity of reactive oxygen species, mitochondrial dysfunction and apoptosis $(7,8)$. The rat bilateral common carotid artery occlusion (BCCAO) model is the most common model used to investigate the effect of chronic cerebral hypoperfusion-induced cognitive dysfunction, and is used to screen drugs with potential therapeutic value for VD (9). Reduced blood flow can result in neuronal energy failure and promote the production of reactive oxygen species, which initiate neuronal apoptosis and lead to the functional deficits typical of VD (10).

Paeoniflorn (PF) (Fig. 1) is the principal bioactive component of Paeoniae radix which has been used for $>1,000$ years in traditional Chinese medicine (11). In modern pharmacological studies, PF exhibits numerous pharmacological effects such as anti-inflammation, antioxidant, endothelium-dependent vasorelaxation, neuromuscular blocking and cognition enhancement properties $(12,13)$. Xiao et al (14) demonstrated that $\mathrm{PF}$ is able to reverse, or alleviate, impairments. such as cerebral infarction, neurological symptoms, tongue protrusion and performance in the water maze, at the chronic stage of transient middle cerebral artery occlusion in rats. Our previous studies have demonstrated that PF significantly attenuates memory impairment in BCCAO model rats (unpublished; Fig. 2) (15); however, the molecular mechanisms underlying the protective effects of PF on VD have not been clearly identified. Therefore, the present study focuses on evaluating 
the neuroprotective effect of $\mathrm{PF}$ and its relation to cell apoptosis in a BCCAO rat model.

\section{Materials and methods}

Animals. Male Sprague Dawley rats (16-18 months old; $300-450$ g body weight) were obtained from the Vital River Laboratories (Beijing, China). Animals were housed with ad libitum access to food and water at a temperature of $22 \pm 2^{\circ} \mathrm{C}$, humidity of $55 \pm 5 \%$ and a 12 -h light/dark cycle. The study was performed in strict accordance with the recommendations in the Guide for the Care and Use of Laboratory Animals of the National Institutes of Health (16). The protocol was approved by the Committee on the Ethics of Animal Experiments of the National Research Institute of Family Planning.

Reagents. PF (purity $>98 \%$ ) was purchased from Shandong Zhongxing Guangrun Biotechnology Co., Ltd. (Nanjing, China). The following primary antibodies were used in western blot analysis: Anti-neuron-specific endolase (NSE) (sc-292097), anti-S100 $\beta$ (sc-28533), anti-brain-derived neurotrophic factor (BDNF) (sc-20981), anti-B-cell lymphoma 2 (Bcl-2) (sc-492), anti-Bcl-2 associated X protein (Bax) (sc-493) and anti-cytochrome $c$ (sc-7159) (all from Santa Cruz Biotechnology, Inc., Dallas, TX, USA).

Study design. PF and saline were administered by oral gavage daily for 28 days. Rats were randomized into the following four groups prior to surgery, according to a computer-generated randomization schedule $(\mathrm{n}=10$ for each group): i) Control group; ii) VD group: $\mathrm{VD}+$ saline ( $2 \mathrm{ml} / \mathrm{kg}$, once daily for 28 days); iii) low dose PF group: VD + PF (20 mg/kg, once daily for 28 days); and iv) high dose PF group: VD + PF (40 mg/kg, once daily for 28 days).

Establishment of vascular dementia. Rats were deeply anesthetized with mebumal sodium $(50 \mathrm{mg} / \mathrm{kg})$ by intraperitoneal injection. A midline incision was made to expose the bilateral common carotid arteries. The common carotid arteries were carefully separated from the surrounding tissues, including the vagus nerve, and ligated with 4-0 silk suture (Johnson\&Johnson Medical Ltd., Wokingham, UK). The control rats were subjected to the same surgical procedure without occlusion of the arteries. During surgery, rectal temperatures were maintained at $37 \pm 0.5^{\circ} \mathrm{C}$ with a thermostatically controlled warming plate (Harvard Apparatus, Holiston, MA, USA).

Collection and preservation of brain tissues. After 28 days of BCCAO, the rats were anesthetized with $10 \%$ chloral hydrate $(350 \mathrm{mg} / \mathrm{kg}$, i.p.) and then decapitated. The brain was rapidly removed and dissected on ice to obtain the hippocampus. All brain tissues were stored at $-80^{\circ} \mathrm{C}$ until further biochemical analysis.

Western blot analysis. The isolated hippocampus were homogenized in lysis buffer [10 mM Tris (Tocris Bioscience, Bristol, UK) (pH 7.4), $100 \mathrm{mM} \mathrm{NaCl}$ (Sinopharm Chemical Reagent Beijing Co., Ltd., Beijing, China),
$1 \mathrm{mM}$ ethylenediamine- $\mathrm{N}, \mathrm{N}, \mathrm{N}^{\prime}, \mathrm{N}^{\prime}$-tetraacetic acid (Tocris Bioscience), $1 \mathrm{mM}$ ethyleneglycol-bis(2-aminoethyl)$\mathrm{N}, \mathrm{N}, \mathrm{N}$, N'-tetraacetic acid (Tocris Bioscience), $1 \mathrm{mM} \mathrm{NaF}$ (Sinopharm Chemical Reagent Beijing Co., Ltd.), $20 \mathrm{mM}$ $\mathrm{Na}_{4} \mathrm{P}_{2} \mathrm{O}_{7}$ (Sinopharm Chemical Reagent Beijing Co., Ltd.), $2 \mathrm{mM} \mathrm{Na}_{3} \mathrm{VO}_{4}$ (Sinopharm Chemical Reagent Beijing Co., Ltd.)], $0.1 \%$ sodium dodecyl sulfate (SDS), $0.5 \%$ sodium deoxycholate (Sinopharm Chemical Reagent Beijing Co., Ltd.), $1 \%$ Triton-X 100 (Amresco LLC, Clevelan, OH, USA), $10 \%$ glycerol (Sinopharm Chemical Reagent Beijing Co., Ltd.), $1 \mathrm{mM}$ phenylmethylsulfonyl fluoride (made from a $0.3 \mathrm{M}$ stock in dimethylsulfoxide; Tocris Bioscience), $60 \mu \mathrm{g} / \mathrm{ml}$ aprotinin (Santa Cruz Biotechnology Inc.), $10 \mu \mathrm{g} / \mathrm{ml}$ leupeptin (Santa Cruz Biotechnology Inc.), and $1 \mu \mathrm{g} / \mathrm{ml}$ pepstatin (Santa Cruz Biotechnology Inc.)] for 30 $\min$. The soluble fraction was obtained by centrifugation at $3,000 \mathrm{x}$ g for $10 \mathrm{~min}$. Protein concentration was determined using a bicinchoninic acid protein assay (Pulilai, Beijing, China). Equal amounts of protein $(40 \mu \mathrm{g})$ were boiled at $100^{\circ} \mathrm{C}$ for $10 \mathrm{~min}$ in loading buffer (Fermentas, Beijing, China) and were separated in $8-10 \%$ SDS-polyacrylamide gel, and electrotransferred onto a polyvinylidene difluoride membrane (Bio-Rad, Hercules, CA, USA). The membrane was blocked with $5 \%$ non-fat milk in $1 \mathrm{X}$ Tris-buffered saline [TBS; $10 \mathrm{mM}$ Tris-HCl (Tocris Bioscience and Sinopharm Chemical Reagent Beijing Co. Ltd.) (pH 8.0), $150 \mathrm{mM} \mathrm{NaCl}$ (Sinopharm Chemical Reagent Beijing Co., Ltd.)] and 0.1\% Tween-20 (TBST; Santa Cruz Biotechnology Inc.) at $25^{\circ} \mathrm{C}$ for $1 \mathrm{~h}$ and subsequently incubated overnight at $4^{\circ} \mathrm{C}$ with the following primary antibodies: Anti-NSE (1:500), anti-S100 $\beta$ (1:500), anti-BDNF (1:500), anti-Bcl-2 (1:1,000), anti-Bax $(1: 1,000)$ and anti-cytochrome $c(1: 1,000)$. The membranes were then washed twice with TBST and probed with the corresponding secondary antibodies conjugated with horseradish peroxidase (sc-2004/sc-2005; HRP; 1:5,000; Santa Cruz Biotechnology Inc.) (anti-mouse/rabbit-HRP was used at a dilution of $1: 5,000$ ) at $25^{\circ} \mathrm{C}$ for $1 \mathrm{~h}$. Following the washes, the blots were developed using an enhanced chemiluminescence system (Amersham ECL plus, GE Healthcare Bio-Sciences, Pittsburgh, PA, USA). The bands were visualized by exposure to X-ray film (X-Omat films, Kodak, Rochester, NY, USA). Anti- $\beta$-actin antibody (sc-130656; 1:2,000; Santa Cruz Biotechnology Inc.) was used as a loading control. All samples were analyzed at least in triplicate.

Statistical analysis. The Morris water maze (15) was used to evaluate spatial memory functioning following the treatment. As described in the previous study (17), the water maze apparatus was a circular pool $(50 \mathrm{~cm}$ height, $150 \mathrm{~cm}$ diameter) with a black inner wall, which was filed with water to $28 \mathrm{~cm}$ and maintained at $22-25^{\circ} \mathrm{C}$. The pool was divided into four quadrants (I-IV) according to four equal distance points on the inner wall. Furthermore, an escape platform painted black ( $26 \mathrm{~cm}$ height, $15 \mathrm{~cm}$ diameter) was submerged $2.0 \mathrm{~cm}$ under the water surface. The rats were given 4 trials per day for 4 days. The trials began as the rat was placed in the pool facing the side wall at a start position and ended once the animal found the platform. In cases where the rat did not find the platform within $90 \mathrm{sec}$, it was guided there. 
Following a period of $20 \mathrm{sec}$ on the platform, the rat was immediately placed again in the pool at a different starting position for the next trial. The swimming traces of the rats were recorded by a camera suspended over the center of the pool. The escape latency and swimming distance of the rats were monitored by a computerized tracking system (Chinese Academy of Medical Sciences, Beijing, China).

The Morris water maze was performed with repeated measures and analyzed by two-way analysis of variance followed by a Bonferroni multiple group comparison. Statistical analysis of other data was performed by one-way analysis of variance followed by a post-Tukey test. For all statistical analyses, a standard software package (Statistical Analysis Software, version 10.0; SAS Institute, Cary, NC, USA) was used. All data are presented as the mean \pm standard error. $\mathrm{P}<0.05$ was considered to indicate a statistically significant difference.

\section{Results}

PF prevents NSE and S100 $\beta$ activation in the hippocampus. A substantial increase in the expression of NSE and S100 $\beta$ activity following VD has been reported. The results of the present study demonstrated that NSE and S100 $\beta$ were significantly increased in the VD group compared with the control group. PF (20 and $40 \mathrm{mg} / \mathrm{kg}$ ) significantly reversed this increase in expression of NSE and S100 $\beta$ in the VD group $(\mathrm{P}<0.05)$ (Fig. 3).

PF regulates apoptosis-related protein expression in the hippocampus. The effects of PF treatment on VD-induced apoptosis-related proteins were examined. The expression levels of Bax and cytochrome $c$ were significantly increased in the VD group compared with the control group $(\mathrm{P}<0.01)$, and this was significantly reversed by treatment with PF (20 and $40 \mathrm{mg} / \mathrm{kg} ; \mathrm{P}<0.05$ ). In addition, the expression level of $\mathrm{Bcl}-2$ was significantly decreased in the VD group compared with the control group $(\mathrm{P}<0.01)$, and treatment with PF (20 and $40 \mathrm{mg} / \mathrm{kg}$ ) significantly reversed this decrease in expression level $(\mathrm{P}<0.05)$ (Fig. 4).

PF upregulates BDNF expression levels in the hippocampus. BDNF protein expression levels in the hippocampus were quantified using western blotting (Fig. 5). The results demonstrated that the BDNF expression level in the VD group was significantly reduced compared with the control group. Treatment with PF (20 and $40 \mathrm{mg} / \mathrm{kg}$ ) significantly reversed this decrease in BDNF expression level $(\mathrm{P}<0.05)$.

\section{Discussion}

Cognitive impairment is a key feature of VD. The hippocampus is highly associated with learning and memory, and is one of the brain regions that is most sensitive to hypoxia and reactive oxygen species. Therefore, the hippocampus is thought to be the primary target brain region of BCCAO-induced damage (18). In the present study, the effects of PF on molecular changes in the hippocampus of $\mathrm{BCCAO}$ rats was investigated. The results demonstrated that administration of PF for 28 days significantly decreased the

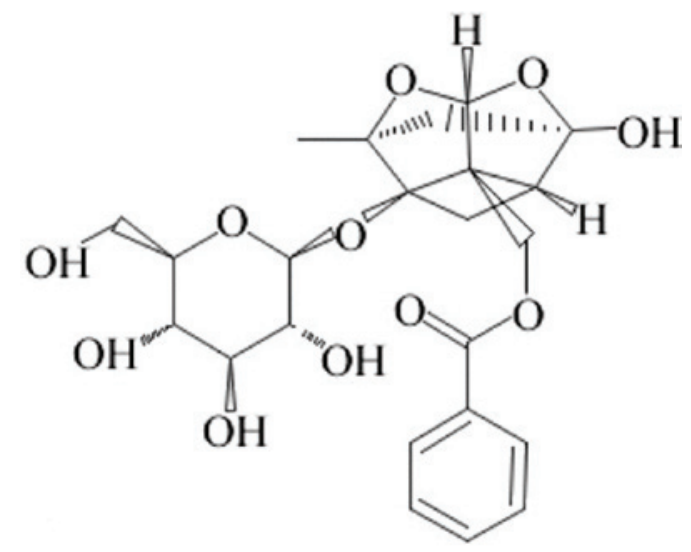

Figure 1. Chemical structure of paeoniflorin.

expression levels of NSE and S100 $\beta$, both sensitive markers for brain damage, in VD model rats. In addition, PF inhibited the initiation of apoptotic cell death, and attenuated the decreased expression of BDNF induced by BCCAO damage. These results strongly support the potential therapeutic role of $\mathrm{PF}$ in VD.

NSE is a cytoplasmic glycolytic enzyme in neurons, and is passively released into the extracellular space under pathological conditions (19). S100 $\beta$ is a calcium-binding protein primarily expressed and secreted by astrocyte cells in the central nervous system; acute elevation of extracellular S100 $\beta$ has been observed in injury conditions (20). NSE and $\mathrm{S} 100 \beta$ expression levels have been considered markers of neurodegeneration and are thought to be associated with the severity of the disease $(21,22)$. The changes in NSE and $\mathrm{S} 100 \beta$ expression levels in the hippocampus of rats were examined in present study. Experimental data showed that the expression levels of NSE and S100 $\beta$ proteins were significantly decreased in PF-treated VD rats compared with VD rats, which indicates that $\mathrm{PF}$ treatment can reverse hippocampus impairment.

Apoptosis is a mode of cell death, and has been proposed to explain the cell loss observed in numerous neurological disorders, including VD (23). Bcl-2 and $B a x$ are two primary genes responsible for the regulation of apoptotic cell death, and their individual products possess opposing functions (24). Bcl-2 is functionally characterized as the apoptosis-suppressing factor, whereas Bax is considered as the apoptosis-promoting factor (25). Bcl-2 inhibits cytochrome $c$ release from mitochondria elicited by the pro-apoptotic molecule Bax, resulting in the inhibition of caspase activation and apoptotic death $(26,27)$. In the present study, treatment with PF significantly reduced the expression levels of Bax and cytochrome $c$, and increased the expression levels of Bcl-2 in the hippocampus of rats with VD. These changes indicate that PF inhibits hippocampal neuron apoptosis in a rat model of VD.

Accumulating evidence has documented the critical role of BDNF, a member of the neurotrophin family, in the regulation of the maintenance, growth and survival of neurons (28). BDNF enhances synaptic transmission and neuronal plasticity in the central nervous system (29), resulting in an increase of learning ability and memory 
$\mathbf{A}$

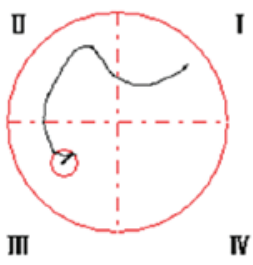

Con

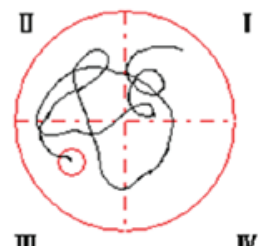

VD

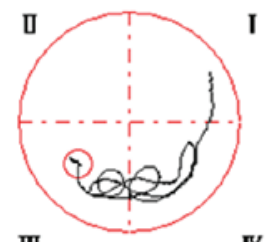

W

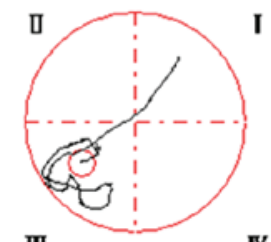

III
W

PF $20 \mathrm{mg} / \mathrm{kg}+$ VD

B
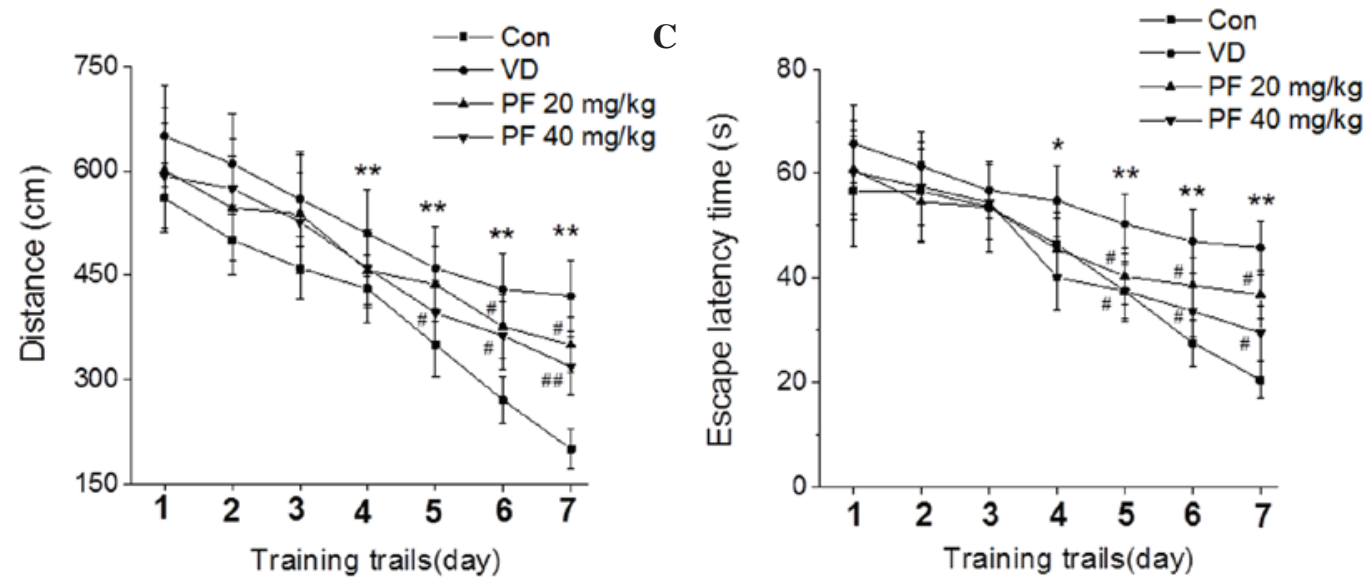

Figure 2. PF attenuates learning and memory impairment in VD rats in the Morris water maze test. (A) Pathway maps of searching for the hidden platform. (B) The distance moved to reach the hidden platform. (C) The mean escape latency time that rats spent finding the hidden platform. ${ }^{*}<<0.05,{ }^{* *} \mathrm{P}<0.01 \mathrm{vs}$. the control group; ${ }^{\#} \mathrm{P}<0.05,{ }^{\# \#} \mathrm{P}<0.05$ vs. the VD group. $\mathrm{n}=7$. Two-way analysis of variance followed by a Bonferroni multiple group comparison were used. Con, control; VD, vascular dementia; PF, paeoniflorn.

A
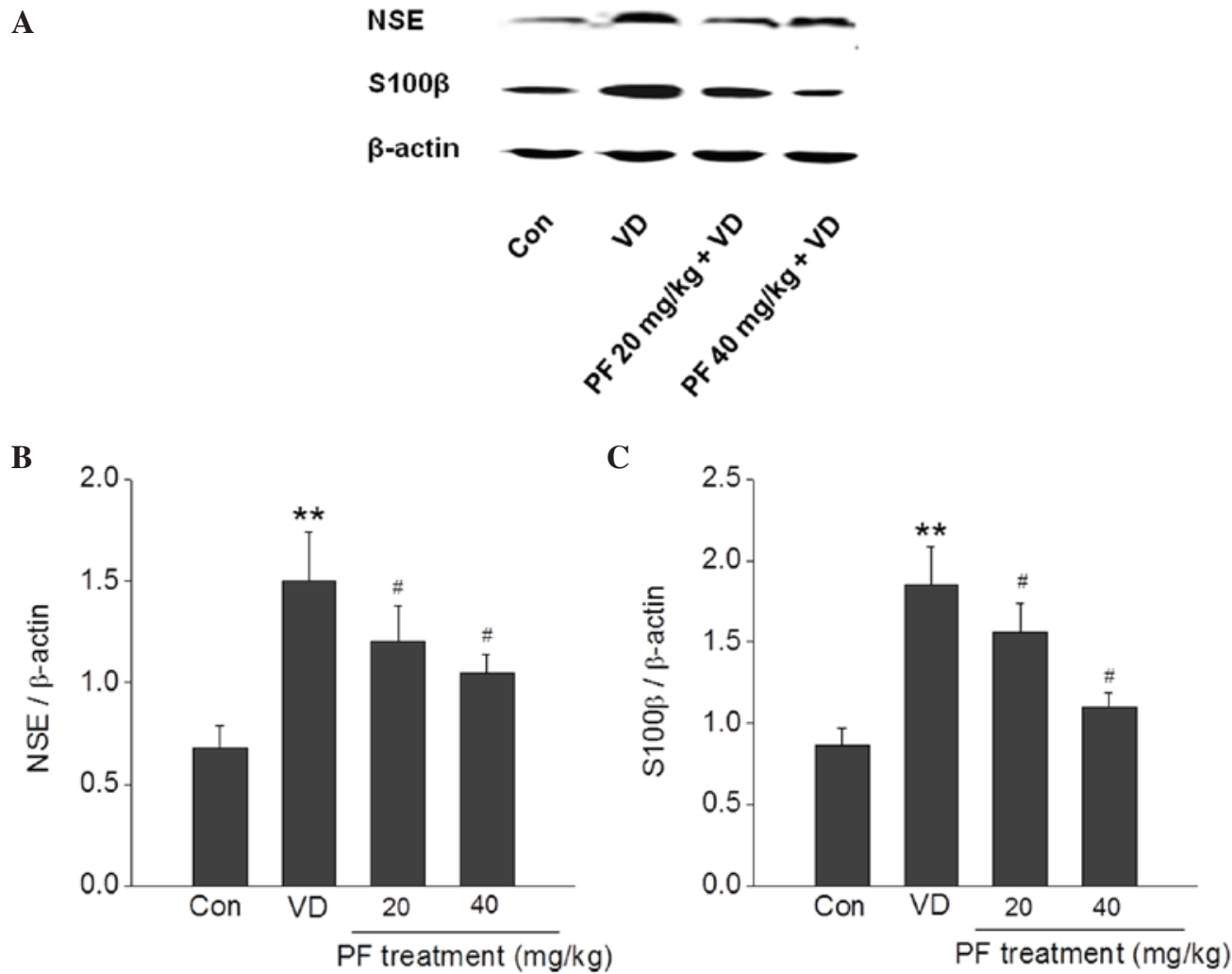

Figure 3. Effects of PF on the protein expression levels of NSE and S100 $\beta$ in the hippocampus of VD rats. (A) Representative images of western blotting showing that PF decreased the expression of NSE and S100 $\beta$. Quantitative analysis of (B) NSE and (C) S100 $\beta$ expression. ${ }^{* *} \mathrm{P}<0.01$ vs. the control group; ${ }^{\#} \mathrm{P}<0.05$ vs. the VD group. $\mathrm{n}=4$. One-way analysis of variance followed by a post Tukey test were used. Con, control; VD, vascular dementia; $\mathrm{PF}$, paeoniflorn. 
A

$$
\text { Bcl-2 }
$$

Bax

Cytochrome c

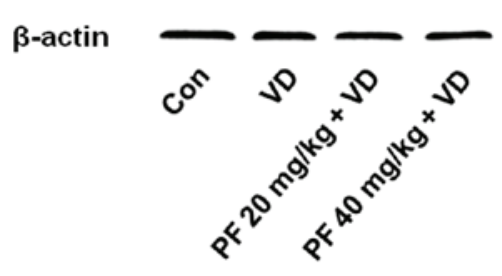

C

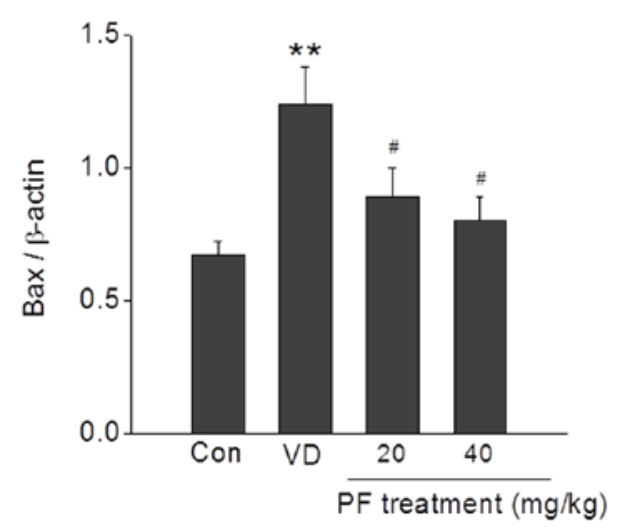

B

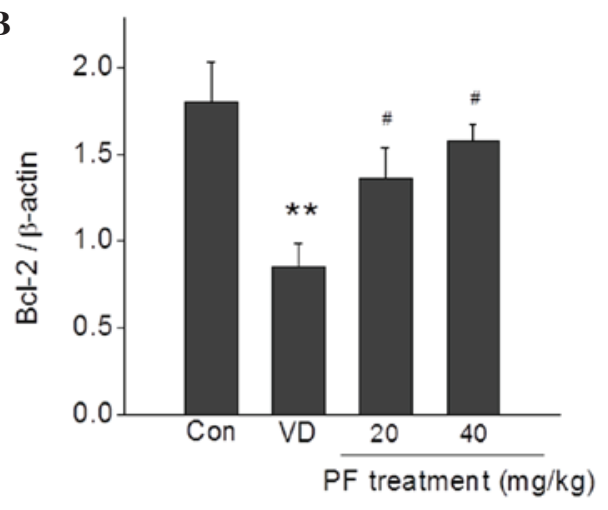

D

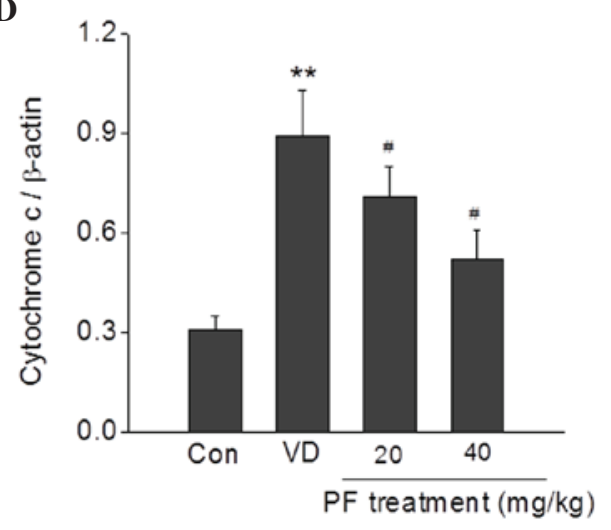

Figure 4. Effects of PF on the expression levels of apoptosis-related proteins in the hippocampus of VD rats. (A) Representative images of western blotting showing that PF decreased Bax and cytochrome $c$ expression levels, and increased Bcl-2 expression levels, in the hippocampus of VD rats. Quantitative analysis of (B) Bcl-2, (C) Bax and (D) cytochrome $c$ expression. ${ }^{* *} \mathrm{P}<0.01$ vs. the control group; ${ }^{*} \mathrm{P}<0.05$ vs. the VD group. $\mathrm{n}=4$. One-way analysis of variance followed by a post Tukey test were used. Bcl-2, B-cell lymphoma 2; Bax, Bcl-2-associated X protein; Con, control; VD, vascular dementia; PF, paeoniflorn.

A

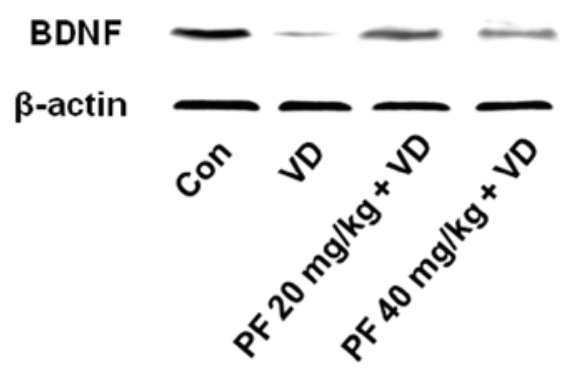

B

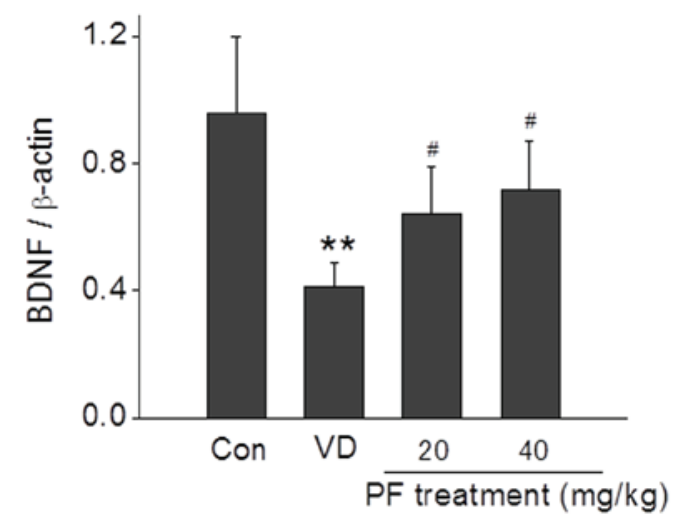

Figure 5. Effects of PF on the protein expression level of BDNF in the hippocampus of VD rats. (A) Representative images of western blotting showing that $\mathrm{PF}$ increased BDNF expression levels. (B) Quantitative analysis of BDNF expression. ${ }^{* *} \mathrm{P}<0.01$ vs. the control group; ${ }^{*} \mathrm{P}<0.05$ vs. the $\mathrm{VD}$ group. $n=4$. One-way analysis of variance followed by a post Tukey test were used. BDNF, brain-derived neurotrophic factor; Con, control; VD, vascular dementia; PF, paeoniflorn. capability (30). BDNF serum concentrations have been reported to correlate with the severity of dementia in patients (31). The results in the current study demonstrated that the neuroprotective effects of PF in VD rats possibly occurred through upregulating the expression of BDNF. BDNF exerts its neuronal protective properties primarily by activating tropomyosin-related kinase B (TrkB) receptors, thus protecting neurons from apoptosis (32). The primary downstream signaling pathways activated by TrkB receptors are phosphatidylinositol 3-kinase/Akt, mitogen-activated protein kinase/Erk or phosphoinositide phospholipase- $\gamma$ signaling pathways. Further studies are required in order to clarify the downstream signaling pathways.

In conclusion, the results from the present study demonstrate that PF produces a protective effect in VD rats by inhibiting the initiation of apoptotic cell death and attenuating the decreased expression of BDNF induced in the VD model. These results confirm the neuroprotective effects of PF on VD and provide a novel insight into the long-term use of $\mathrm{PF}$ as a potential treatment in the early cognitive impairment stages of VD.

\section{Acknowledgements}

The present study was supported by the National Key Grant of Basic Research Project (grant no. 2010CB530403) and the Capital Medical University National Key Discipline Project (grant no. CMUNEURO-201403). 


\section{References}

1. Ronnemaa E, Zethelius B, Lannfelt L and Kilander L: Vascular risk factors and dementia: 40 -Year follow-up of a population-based cohort. Dement Geriatr Cogn Disord 31: 460-466, 2011.

2. Malouf R and Birks J: Donepezil for vascular cognitive impairment. Cochrane Database Syst Rev CD004395, 2004.

3. Ferri CP, Prince M, Brayne C, Brodaty H, Fratiglioni L, Ganguli M, Hall K, Hasegawa K, Hendrie H, Huang Y, et al: Global prevalence of dementia: A Delphi consensus study. Lancet 366: 2112-2117, 2005.

4. Yang S, Zhou GG, Liu H, et al: Portective effects of p38 MAPK inhibitor SB202190 against hippocampal apoptosis and spatial learning and memory deficits in a rat model of vascular dementia. Biomed Res Int, 2013: 215798, 2013.

5. Jellinger KA: The enigma of vascular cognitive disorder and vascular dementia. Acta Neuropathol 113: 349-388, 2007.

6. Levine DA and Langa KM: Vascular cognitive impairment: Disease mechanisms and therapeutic implications. Neurotherapeutics 8: 361-373, 2011.

7. Bennett S, Grant MM and Aldred S: Oxidative stress in vascular dementia and Alzheimer's disease: A common pathology. J Alzheimers Dis 17: 245-257, 2009.

8. Wang J, Zhang HY and Tang XC: Cholinergic deficiency involved in vascular dementia: Possible mechanism and strategy of treatment. Acta Pharmacol Sin 30: 879-888, 2009.

9. Gong X, Ma M, Fan X, Li M, Liu Q, Liu X and Xu G: Down-regulation of IGF-1/IGF-1R in hippocampus of rats with vascular dementia. Neurosci Lett 513: 20-24, 2012.

10. Kasparova S, Brezova V, Valko M, et al: Study of the oxidative stress in a rat model of chronic brain hypoperfusion. Neurochem Int 46: 601-611, 2005.

11. Nizamutdinoval IT, Jim YC, Kim JS, et al: Paconol and paconiflorin, the main active principles of Paconiaalbiflora, protect the heart from myocardial ischemia/reperfusion injury in rats. Planta Med 74:14-18, 2008

12. Watanabe H: Candidates for cognitive enhancer extracted from medicinal plants: Paeoniflorin and tetramethylpyrazine. Behav Brain Res 83: 135-141, 1997.

13. Tang NY, Liu CH, Hsieh CT and Hsieh CL: The anti-inflammatory effect of paeoniflorin on cerebral infarction induced by ischemia-reperfusion injury in Sprague-Dawley rats. Am J Chin Med 38: 51-64, 2010.

14. Xiao L, Wang YZ, Liu J, Luo XT, Ye Y and Zhu XZ: Effects of paeoniflorin on the cerebral infarction, behavioral and cognitive impairments at the chronic stage of transient middle cerebral artery occlusion in rats. Life Sci 78: 413-420, 2005.

15. Zhang LG, Wang LJ, Shen QQ, et al: Paeoniflorin Improves Regional Cerebral Blood Flow and Suppresses Inflammatory Factors in the Hippocampus of Rats with Vascular Dementia. Chin J Integr Med Epub ahead of print.

16. Institute of Laboratory Animal Research, Commission on Life Sciences, National Research Council: Guide for the Care and Use of Laboratory Animals. 7th edition. National Academy Press, Washington, D.C., pp56-66, 1996.

17. D'Hooge R and De Devn PP: Applications of the Morris water maze in the study of learning and memory. Brain Res Brain Res Rev 36: 60-90, 2001.
18. Farkas E, Luiten PG and Bari F: Permanent, bilateral common carotid artery occlusion in the rat: A model for chronic cerebral hypoperfusion-related neurodegenerative diseases. Brain Res Rev 54: 162-180, 2007.

19. Berger RP, Dulani T, Adelson PD, Leventhal JM, Richichi R and Kochanek PM: Identification of inflicted traumatic brain injury in well-appearing infants using serum and cerebrospinal markers: A possible screening tool. Pediatrics 117: 325-332, 2006.

20. Gonçalves CA, Leite MC and Nardin P: Biological and methodological features of the measurement of S100B, a putative marker of brain injury. Clin Biochem 41: 755-763, 2008.

21. Mecocci P, Parnetti L, Romano G, Scarelli A, Chionne F, Cecchetti R, Polidori MC, Palumbo B, Cherubini A and Senin U: Serum anti-GFAP and anti-S100 autoantibodies in brain aging, Alzheimer's disease and vascular dementia. J Neuroimmunol 57: 165-170, 1995.

22. Parnetti L, Palumbo B, Cardinali L, Loreti F, Chionne F, Cecchetti R and Senin U: Cerebrospinal fluid neuron-specific enolase in Alzheimer's disease and vascular dementia. Neurosci Lett 183: 43-45, 1995.

23. Sun ZK, Ma XR, Jia YJ, et al: Effects of resveratrol on apoptosis in a rat model of vascular dementia. Exp Ther Med 7:843-848, 2014.

24. Min JJ, Huo XL, Xiang LY, et al: Protective effect of Dl-3nbutylphthalide on learning and memory impairment induced by chronic intermittent hypoxia-hypercapnia exposure. Sci Rep 4:5555,2014

25. Hwang L, Choi IY, Kim SE, Ko IG, Shin MS, Kim CJ, Kim SH, Jin JJ, Chung JY and Yi JW: Dexmedetomidine ameliorates intracerebral hemorrhage-induced memory impairment by inhibiting apoptosis and enhancing brain-derived neurotrophic factor expression in the rat hippocampus. Int $\mathrm{J}$ Mol Med 31: 1047-1056, 2013.

26. Shimizu S, Narita M and Tsujimoto Y: Bcl-2 family proteins regulate the release of apoptogenic cytochrome c by the mitochondrial channel VDAC. Nature 399: 483-487, 1999.

27. Kluck RM, Bossy-Wetzel E, Green DR and Newmeyer DD: The release of cytochrome $\mathrm{c}$ from mitochondria: A primary site for Bcl-2 regulation of apoptosis. Science 275: 1132-1136, 1997.

28. Chuang DM: The antiapoptotic actions of mood stabilizers: molecular mechanisms and therapeutic potentials. Ann N Y Acad Sci 1053:195-204, 2005.

29. Schinder AF and Poo M: The neurotrophin hypothesis for synaptic plasticity. Trends Neurosci 23: 639-645, 2000.

30. Mizuno M, Yamada K, Olariu A, Nawa $H$ and Nabeshima T: Involvement of brain-derived neurotrophic factor in spatial memory formation and maintenance in a radial arm maze test in rats. J Neurosci 20: 7116-7121, 2000.

31. Laske C, Stransky E, Leyhe T, Eschweiler GW, Maetzler W, Wittorf A, Soekadar S, Richartz E, Koehler N, Bartels M, et al: BDNF serum and CSF concentrations in Alzheimer's disease, normal pressure hydrocephalus and healthy controls. J Psychiatr Res 41: 387-394, 2007.

32. Murer MG, Yan Q and Raisman-Vozari R: Brain-derived neurotrophic factor in the control human brain and in Alzheimer's disease and Parkinson's disease. Prog Neurobiol 63:71-124, 2001. 